NANO: Brief Reports and Reviews

Vol. 8, No. 4 (2013) 1350044 (11 pages)

(C) World Scientific Publishing Company

DOI: $10.1142 /$ S1793292013500446

\title{
SURFACE PLASMON EXCITATION AND MANIPULATION IN DISORDERED TWO-DIMENSIONAL NANOPARTICLE ARRAYS
}

\author{
VICTOR COELLO* and RODOLFO CORTES \\ Centro de Investigación Científica y de Estudios \\ Superiores de Ensenada BC, Unidad Monterrey \\ Km 9.5 Carretera Aeropuerto, Parque de Investigacion e \\ Innovacion Tecnologica (PIIT) Apodaca \\ Nuevo Leon 66629, Mexico \\ *vcoello@cicese.mx \\ CESAR E. GARCIA-ORTIZ and NORA ELIZONDO \\ Universidad Autonoma de Nuevo Leon, Doctorado en Ingenieria \\ Fisica Industrial-FCFM, Pedro de Alba $S / N$ \\ San Nicolas de los Garza, Nuevo Leon 66451, Mexico \\ Received 15 November 2012 \\ Accepted 8 April 2013 \\ Published 14 May 2013
}

\begin{abstract}
We present experimental and numerical results of simultaneous surface plasmon polariton (SPP) excitation and in-plane manipulation with random arrays of gold nanoparticles. The recorded images were obtained by using leakage radiation microscopy (LRM) for the excitation wavelength of $633 \mathrm{~nm}$ and for different densities of particles. The numerical model makes use of a composed analytic Green dyadic which takes into account near- and far-field regions, with the latter being approximated by the part describing the scattering via excitation of SPP. The LRM optical images obtained are related to the calculated SPP intensity distributions demonstrating that the developed approach can be successfully used in studies of systems of closely spaced arrays.
\end{abstract}

Keywords: Surface plasmon polaritons, green dyadic, multiple scattering.

\section{Introduction}

Plasmonics is a very active research area dealing with fundamental studies of surface plasmon polaritons (SPPs), ${ }^{1}$ and opening promising technological perspectives within nano-optics, e.g., in miniaturized photonic components. ${ }^{2}$ SPPs are electromagnetic excitations coupled to electron plasma oscillations, which have the property of propagating along a metal/dielectric interface as quasi twodimensional (2D) interface waves. Even though the phenomenon of SPP has been known for a long time ${ }^{3}$ its local study is relatively recent and has been strongly motivated by the development of imaging techniques such as the scanning near-field optical microscopy (SNOM), ${ }^{4,5}$ and the fluorescence microscopy. ${ }^{6}$ The two techniques each had their own disadvantages. SNOM has a resolution which mostly 
depends on tip quality, and an optical mapping that is relatively slow whereas fluorescence microscopy exhibits molecular photobleaching and therefore does not allow quantitative analysis. An alternative developed recently that overcome these limitations is the so-called leakage radiation microscopy (LRM). ${ }^{7}$ The technique is simple in conception and provides a rapid 2D SPP mapping, and the possibility of simultaneous access to direct and reciprocal space. LRM has stimulated plasmonics research in various directions, one of them being SPP interaction and manipulation with nanostructures. In this context, the interaction of SPP waves with nanostructures has been investigated ranging from individual nanometer-sized film structures, ${ }^{7}$ including quantum dots, ${ }^{8}$ to more complex plasmonic elements such as Bragg mirrors,${ }^{9}$ beam splitters, ${ }^{10}$ waveguides, ${ }^{11,12}$ refracting elements, ${ }^{13}$ etc. In general, the aforementioned phenomena are related to the propagation of SPP waves through periodic or nanostructured plasmonic elements. Considering the SPP interaction with randomly 2D nanoparticles arrays, extensive theoretical studies have been conducted. ${ }^{14-16}$ However, experimental evidences of related phenomena such as localization ${ }^{17}$ and photon bandgap effect of SPPs $^{18}$ are still scarce. The experimental framework is not trivial since the unwanted process of SPP scattering into a free space is always present during the course of SPP propagation. Those radiative losses increase as the volume fraction of nanoparticles becomes larger, and as a consequence rather complicated and misleading field patterns may appear in the surface plane. On the other hand, a relatively large density of nanoparticles is necessary in order to affect the transport of the light, and therefore, localize electromagnetic modes in standing rather than travelling waves. ${ }^{17}$ This suggests a trade-off that needs to be explored further. In this context, using a vectorial dipolar model for multiple SPP scattering, ${ }^{19}$ we have numerically demonstrated the possibility of simultaneous SPP excitation and in-plane manipulation with square-lattice arrays of nanoparticles. ${ }^{20}$ The main idea was in avoiding the usage of local SPP excitation elements as for example, incoupling ridges, ${ }^{21}$ subwavelength hole arrays on a thick metal film, ${ }^{22,23}$ or nanotubes. ${ }^{24}$ Therefore, it seems plausible that reducing the number of components could help not only in the miniaturization of plasmonics circuits but also in minimizing unwanted SPP scattering and interference effects. Recently, we extended the aforementioned approach, by introducing a composed analytic Green dyadic which takes into account near- and far-field regions, and used to calculate simultaneous SPP excitation and in-plane propagation inside square-random arrays of nanoparticles. ${ }^{25}$ The composed Green dyadic represented an improvement of previous SPP simulations for random nanoparticles arrays since it permitted SPP scattering simulations for more realistic systems with relatively large number of close, or even in contact, nanoparticles. A system of closely spaced arrays of nanoparticles is desirable in, for example, plasmonic field enhancement for sensitive detection of biological samples. ${ }^{26}$

Here, using a LRM, we experimentally investigate the possibility of simultaneous SPP excitation and propagation control in random $2 \mathrm{D}$ arrays of nanoparticles illuminated by a normally incident Gaussian beam. We compare our optical images with the previously reported ${ }^{25}$ and the additionally obtained numerical results. The paper is organized as follows. In Sec. 2, the main relationships used in our calculations are given and explained. The experimental setup and the techniques for sample preparation, in general terms, are presented in Sec. 3. Typical experimental LRM images of the SPP excitation by nearly isolated particles are presented in Sec. 4. In Sec. 5, numerical and experimental images of several regimes of multiple scattering are presented for randomly situated nanoparticles and the correlation between the regimes and their corresponding spatial Fourier spectra are discussed. In Sec. 6, a simple optical refracting system composed of randomly situated nanoparticles is considered. Finally, in Sec. 7, we summarize the results obtained and offer our conclusions.

\section{The Model}

The model is based on the assumptions that the inplane SPP scattering is dominant with respect to the SPP scattering out of the plane, and that if the light is incident on a metal/dielectric interface with scattering objects, the objects can be modeled as point-like dipoles. ${ }^{25}$ For out of plane scattering of SPPs, we will consider propagating field components scattered away from the surface decreasing the total energy stored in SPPs. In-plane scattering of SPPs occurs when SPPs are scattered by surface imperfections along the surface plane, i.e., into other SPPs preserving the total SPP energy. These assumptions led to the construction of an approximate Green's 
tensor describing SPP-to-SPP scattering by a dipolar point-like nanoparticle located at a metal/ dielectric interface. The validity of the model has been established for relatively large inter-particle distances, whereas for smaller distances it is more accurate to use a composed analytic Green dyadic which takes into account near- and far-field regions (SPP to SPP scattering) with the first being approximated by the near-field electrostatic approximation of the total Green dyadic. ${ }^{25}$

The self-consistent polarization of each scatterer established in the process of multiple scattering is obtained by solving the following equation:

$$
\mathbf{P}_{i}=\boldsymbol{\alpha}_{i} \cdot \mathbf{E}^{0}\left(\mathbf{r}_{i}\right)+\frac{k_{0}^{2}}{\varepsilon_{0}} \sum_{n \neq i} \boldsymbol{\alpha}_{i} \cdot \mathbf{G}\left(\mathbf{r}_{i}, \mathbf{r}_{n}\right) \cdot \mathbf{P}_{n}
$$

where $\mathbf{P}_{i}$ is the polarization of the particle $i, \alpha_{i}$ is the polarizability tensor for particle $i$ with the multiple scattering between the particle and the metal surface taken into account (surface dressing effect), $\mathbf{E}^{0}$ is an incoming electric field, $k_{0}$ is the free space wave number, $\varepsilon_{0}$ is the vacuum permittivity and $\mathbf{G}\left(\mathbf{r}_{i}, \mathbf{r}_{n}\right)$ is the Green's tensor for the reference structure (total field propagator). The Green's tensor $\mathbf{G}$ is the sum of a direct contribution $\mathbf{G}_{d}$, in this case the free space Green's tensor, and an indirect contribution $\mathbf{G}_{S}$ that describes both reflection from the metal/dielectric interface and excitation of SPPs. In order to take into account spatial distribution of the incident field, which interacts with the particles, we assume that the incoming electric field is determined by the following expression $\mathbf{E}^{0}=\hat{\mathbf{e}} \exp \left(-\left[x^{2}+y^{2}\right] / W^{2}\right)$, where $\hat{\mathbf{e}}$ is the unit polarization vector and $W$ is the waist of the incident light beam. In a general form, $\mathbf{E}^{0}$ should be a field for the reference geometry, which would include the reflection from the surface and a phase difference between the incident and the reflected fields at a height $z$ above the surface. This will in practice only result in a scaling factor and therefore one can use the above expression for $\mathbf{E}^{0}$ to describe the illumination conditions with reasonably accurate results. For a spherical particle made of the same metal as the substrate, the polarizability tensor is given $b^{28-30}$ :

$$
\alpha \approx\left[\mathbf{I}-\frac{\varepsilon-1}{\varepsilon+1} \frac{\varepsilon-1}{\varepsilon+2}\left(\frac{1}{8} \hat{x} \hat{x}+\frac{1}{8} \hat{y} \hat{y}+\frac{1}{4} \hat{z} \hat{z}\right)\right]^{-1} \cdot \alpha^{0},
$$

where $\mathbf{I}$ is the unit dyadic tensor, $\varepsilon$ is the metal dielectric constant, $\hat{x}, \hat{y}, \hat{z}$ are unit vectors in a Cartesian coordinate system with $\hat{z}$ being perpendicular to the air-metal interface, and $\alpha^{0}=$ $\varepsilon_{0} \mathbf{I} 4 \pi a^{3 \frac{\varepsilon-1}{\varepsilon+2}}$ is the free space polarizability tensor in the long-wave electrostatic approximation with $a$ being the sphere radius. The polarizations [see Eq. (1)] and the total field,

$$
\mathbf{E}(\mathbf{r})=\mathbf{E}^{0}(\mathbf{r})+\frac{k_{0}^{2}}{\varepsilon_{0}} \sum_{n} \mathbf{G}\left(\mathbf{r}, \mathbf{r}_{n}\right) \cdot \mathbf{P}_{n},
$$

can be calculated using the appropriate Green's tensor for the reference structure $\mathbf{G}\left(\mathbf{r}, \mathbf{r}^{\prime}\right)$. Finally, based on the initial assumptions, we have used the analytic representations of the Green dyadic in the near- and far-field (SPP to SPP propagator) regions. The complete analysis of the validity domain of such an approximation as well as the suitable limit to distinguish between the uses of these expressions were presented in detail elsewhere. ${ }^{25}$

\section{Experimental Techniques}

\subsection{Sample preparation}

In order to prepare the sample, a small drop containing a water solution of colloidal gold nanoparticles (spheres) was then deposited on a gold thin film $(50 \mathrm{~nm})$, which was previously thermally evaporated on a glass substrate. The drop evaporates after some minutes leaving a high-density circular region with gold nanoparticles. When a drop of colloidal solution of nanoparticles dries on a surface it leaves behind a stain-like ring ("coffee stain") of material with clumps of particles in the interior and a few number of them on the outside of the ring. Thus, the final sample structure consisted of both a high- and a low-density scattering regions composed of gold bumps randomly distributed over the thin gold film [see Figs. 1(a)-1(c)]. The scattering regions densities were approximately of 10 (low) and 50 (high) particles per $1 \mu \mathrm{m}^{2}$. The colloidal gold nanoparticles were synthesized by using the green chemical method which is a simple, cost effective and environmental friendly technique (more information about our procedure is found in Ref. 27). The transmission electron and atomic force microscopy studies revealed an average particles size distribution ranging from $20 \mathrm{~nm}$ to $100 \mathrm{~nm}$ [see Fig. 1(a)-1(c)]. 


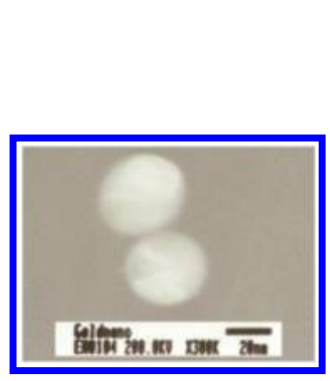

(a)

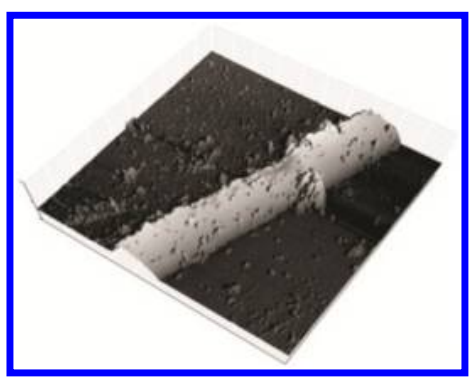

(b)

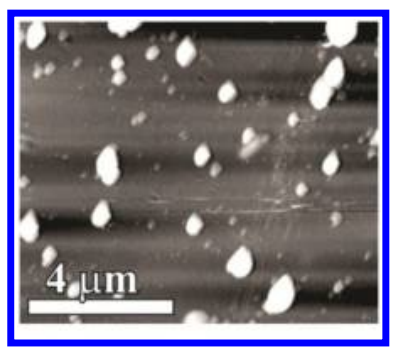

(c)

Fig. 1. (a) TEM image of gold nanoparticles synthesized by the green synthesis method. AFM images $\left(65 \times 65 \mu \mathrm{m}^{2}\right)$ of the gold sample surface showing (b) a high- and a low-density scattering regions composed of gold bumps randomly distributed and $(\mathrm{c})$ zoom $\left(10 \times 10 \mu \mathrm{m}^{2}\right)$ on the low-density area showing individual particles.

\subsection{Leakage radiation microscopy}

The experimental setup used for SPP imaging is shown in Fig. 2. SPPs are excited locally by focusing polarized light from a $\mathrm{He}-\mathrm{Ne}$ laser $(633 \mathrm{~nm})$ through a $20 \times(\mathrm{NA}=0.4)$ microscope objective (O1) onto the surface of the artificially nanostructured gold film (S). The image of leakage radiation is collected on a CCD camera after passing through a $63 \times(\mathrm{NA}=1.25)$ oil-immersion objective (O2). The system allows one to study SPP propagation in both the direct and indirect space (Fourier space, $\varphi$ ). Imaging Fourier space is possible by recording the LRM field in the back focal plane of the oil immersion objective. A filter (BB) in Fourier space may be used to help that only the waves corresponding to LRM are resolved, in order to maximize the CCD image contrast and reduce noise. To facilitate observation of the surface structure, a lamp illumination is conjugated with the $20 \times$ objective. A neutral density filter (NDF) was used to attenuate the probe laser intensity in order to avoid saturation in the CCD camera.

\section{Random Particles Arrays: Low-Density Area}

First we imaged the LRM field created by an incident (linear polarized) Gaussian beam, $x$-pol, $\lambda=633 \mathrm{~nm}, \mathrm{FWHM}=2 \mu \mathrm{m}$, and impinging on a small area of the low-density region of nanoparticles. Hereafter all the images are presented in linear grayscale. Two extended lobes similar to those related to dipole-like damped radiation from single nanoparticles are clearly seen in the direct space image [see Fig. 3(a)]. This is achieved by focusing the illumination beam to a cluster of a few particles since our imaging system cannot provide enough information of spatial resolution about the position of a single nanoparticle [see Fig. 3(b)]. Such SPP beam scattering was numerically simulated [see Fig. 3(c)] using the total Green's tensor formalism described in Sec. 2. Hereafter, the entire systems are simulated on a gold surface with dielectric constant $\varepsilon=-11+1.4 i$ and the illumination conditions are kept the same for all the calculations. The calculated images show grayscale representations of the distributions of the intensity $|E|^{2}$ for the illumination wavelength $(633 \mathrm{~nm})$. The total intensity field was calculated $80 \mathrm{~nm}$ above the air-gold interface, and the incident beam has been removed; i.e., only scattered SPP appear in the pictures. The configuration and the illumination conditions can be considered as fairly similar to the experimental ones, for example we have used a cluster system of 10 particles. The radius of the particles $(30 \mathrm{~nm})$ is a fitting parameter which is chosen to match the experimental images. This is because the size of the particles used in simulations is only relevant within

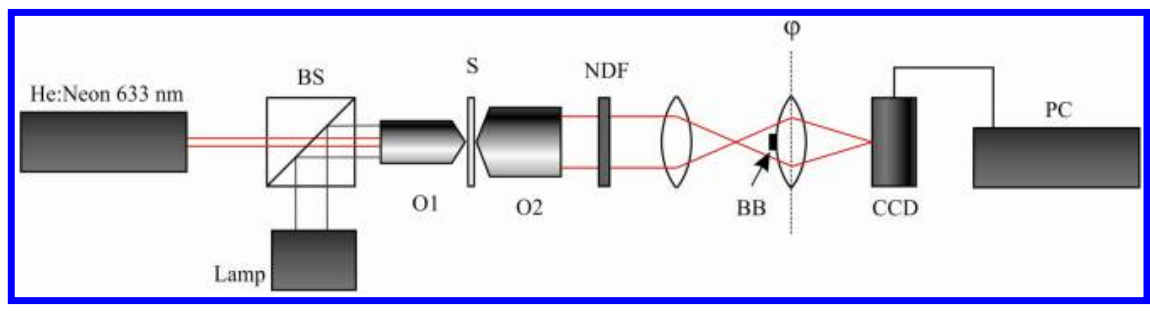

Fig. 2. Experimental setup for LRM. 


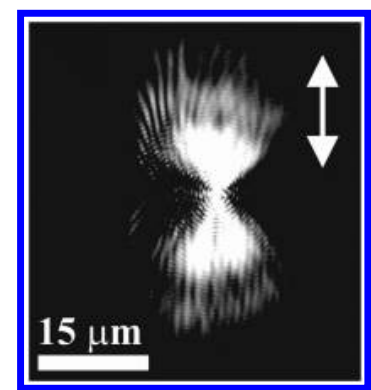

(a)

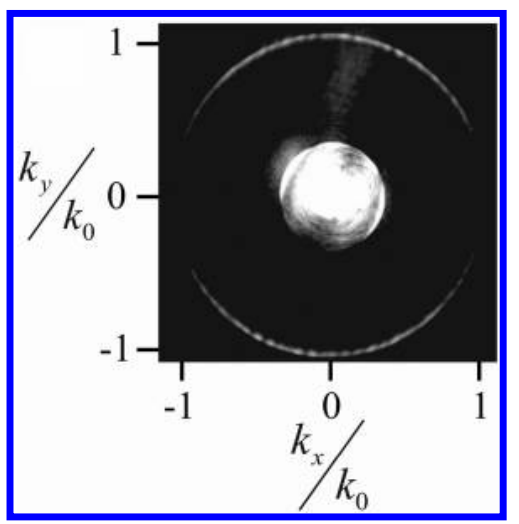

(d)

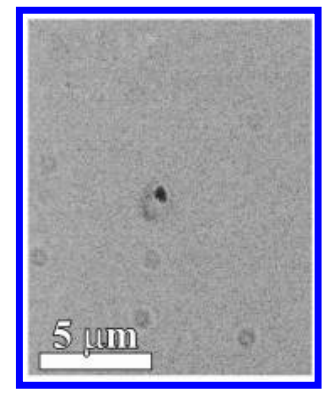

(b)

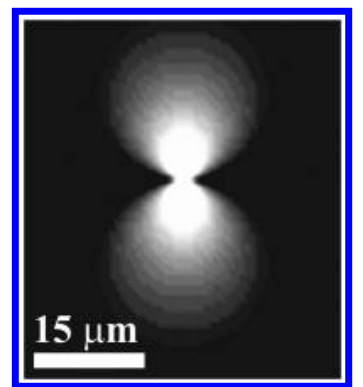

(c)

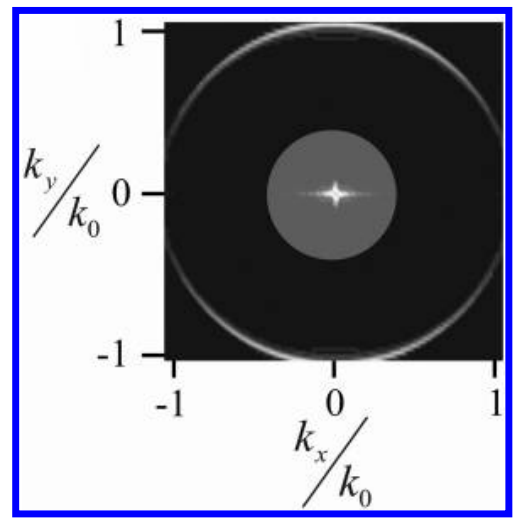

(e)

Fig. 3. (a) LRM of a dipole-like damped radiation. (b) Topographic image of a small nanoparticle cluster. (c) Dipole-like damped radiation calculated image. Fourier space (d) LRM and (e) calculated images of (a) and (c), respectively. The arrow indicates the incident light polarization in (a) and (c).

the model and influences the polarizability of the particles, ${ }^{19,25}$ but does not bear a direct relation to the size of the scatterers in the experiment. The leakage radiation recorded in the Fourier plane of the microscope [see Fig. 3(d)] exhibits two crescents that are characteristic of the $\mathrm{Au} / \mathrm{Air}$ SPP mode, and which also indicate the polarization direction. ${ }^{31}$ In the calculated Fourier spectrum of Fig. 3(e), one can see a very good agreement with the main features of Fig. 3(d). In order to get a more realistic numerical calculation, the numerical aperture of the collection objective was taken into account, i.e., in our calculations, the Fourier image is limited to $\sqrt{k_{x}^{2}+k_{y}^{2}} / k_{0} \leq \mathrm{N}$.A. The field distribution displayed in Fig. 3(a) mainly shows the field component parallel to the polarization of the incident field. However, the horizontal orthogonal component though being weaker is different from zero. In order to map such component and therefore to study, in more detail, the near- and far-field scattering contributions, we have performed LRM measurements in cross polarized detection. For this reason, a linear polarizer was placed in front of the CCD camera of our experimental setup. The intensity distributions of the horizontal component being dictated by nondiagonal elements of the field propagators may produce complicated patterns. In accordance with this, now we observed a four-lobe pattern in both the recorded [see Fig. 4(a)] and calculated images [see Fig. 4(b)]. Based on the proposed model, one should expect to distinguish a transition zone in the obtained pattern since we use a near-field dyadic for distances shorter and a SPP dyadic for distances longer than a specified fraction of the illumination light wavelength. ${ }^{25}$ The central part of the calculated image clearly exhibited that the extended lobes are formed only after an initial transition region [see Fig. 3(d)]. A similar transition between bright spots and elongated lobes is evident in the experimental obtained image [see Fig. 3(c)]. The field distribution displayed in Fig. 3(a) mainly shows the field component parallel to the polarization of the incident field. However, the horizontal orthogonal component though being weaker is different from zero. In order to map such component 


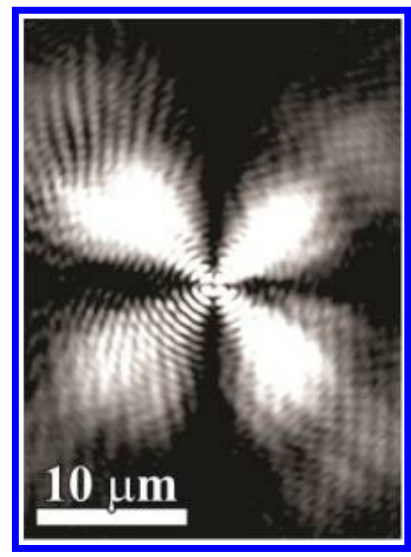

(a)

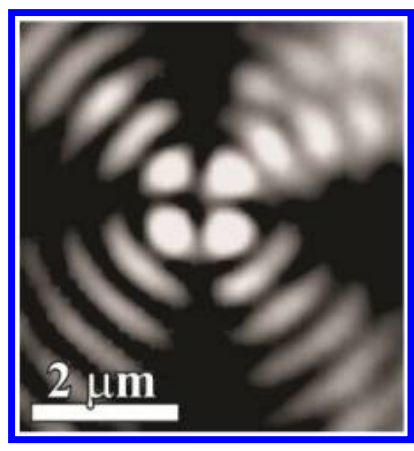

(c)

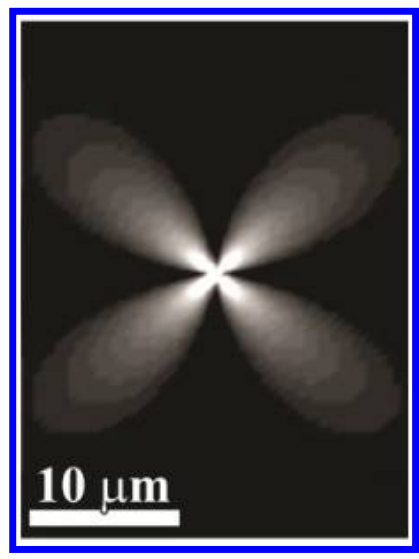

(b)

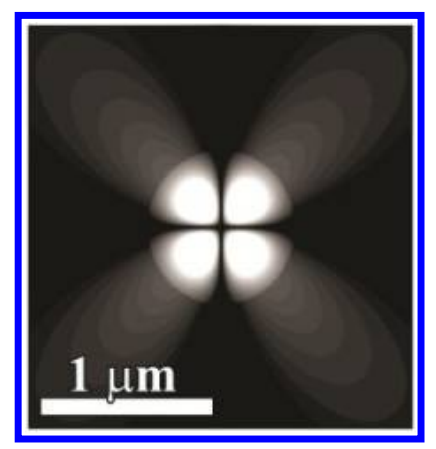

(d)
Fig. 4. Cross polarized (a) LRM and (b) calculated images of a dipole-like radiation. (c) and (d) Zoom of the central part in (a) and (b), respectively. Incident light polarization is the same as in Fig. 3.

and therefore to study, in more detail, the near- and far-field scattering contributions, we have performed LRM measurements in cross polarized detection. For this reason, a linear polarizer was placed in front of the CCD camera of our experimental setup. The intensity distributions of the horizontal component being dictated by nondiagonal elements of the field propagators may produce complicated patterns. In accordance with this, now we observed a four-lobe pattern in both the recorded [see Fig. 4(a)] and calculated images [see Fig. 4(b)]. Based on the proposed model, one should expect to distinguish a transition zone in the obtained pattern since we use a near-field dyadic for distances shorter and a SPP dyadic for distances longer than a specified fraction of the illumination light wavelength. ${ }^{25}$ The central part of the calculated image clearly exhibited that the extended lobes are formed only after an initial transition region [see Fig. 4(d)]. A similar transition between bright spots and elongated lobes is evident in the experimentally obtained image [see Fig. 4(c)].
A point dipole emitter not only excites a SPP, but also has its own dipolar emission and its diffraction pattern (similar to the one generated by a circular aperture) that overlay coherently on the leakage radiation image of the SPP. ${ }^{7}$ The interference of these contributions, as well as the finite numerical aperture of the collection objective, lead to strong circular fringes at the image center of Fig. 4(c). Regarding the theoretical calculation, as it was mentioned in the total field calculations the incident beam has been removed; i.e., only scattered SPP appear in the pictures and therefore the experimentally observed interference pattern is not present. Insets (a) and (b) of Fig. 5 show a direct SPP excitation taking place at the center of a linelike surface structure with a certain inclination angle with respect to axis light polarization. We think that such line is formed, in the low density area, by a short linear chain of the Au nanoparticles [see Fig. 5(a)]. The light-SPP coupling of Fig. 5(b) strongly resembles the classical coupling through a ridge structure $^{21}$ where the process results in a slightly diverging SPP beams propagating away from the ridge. Figure 5(c) shows the result of the corresponding modeling. There, as an incident wave, we used a Gaussian beam having the waist situated at the center of a 150 -nm-period line of nanoparticles (length $=2.5 \mu \mathrm{m}$ ). In a previous work, ${ }^{20}$ we demonstrated that a similar array works efficiently with a period of $150 \mathrm{~nm}$. However, one should establish that as long as the array period is sufficiently smaller than the incident SPP wavelength, we consider this calculation method as a one giving the correct result, provided that the size of the particles is adjusted appropriately. The line-like structure has an angle orientation similar to that observed in the experimental image. The SPP excitation is evidenced by the SPP beam coming out of the line-like structure [see Figs. 5(b) and 5(c)], and by the corresponding Fourier transform [see Figs. 5(d) and 5(e)]. Here, it is important to note that not all the synthesized nanoparticles were perfectly spherical. Bearing this fact in mind, in the corresponding experimental real space image, one should expect that the SPP propagation leads to a slightly asymmetric double lobe structure [see Fig. 5(b)]. The same characteristics of plasmon excitation are seen in the corresponding Fourier space image [see Fig. 5(d)]. In the ideal numerical case, the point-like particles are assumed perfect symmetric [see Fig. 5(c)] and therefore one can observe 


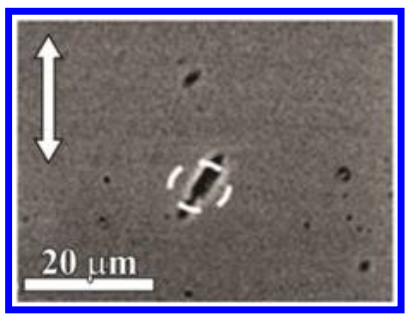

(a)

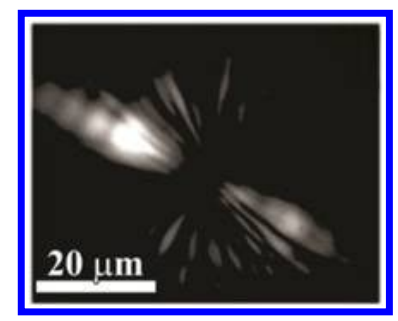

(b)

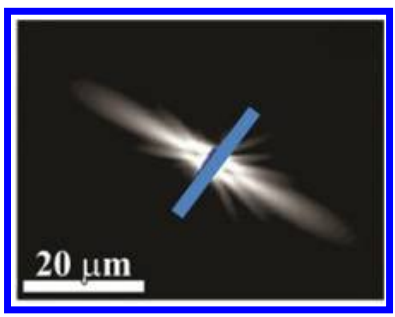

(c)

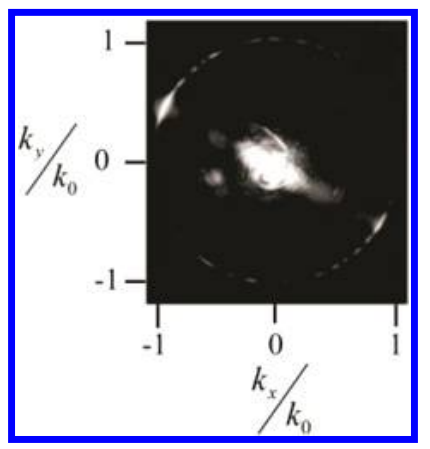

(d)

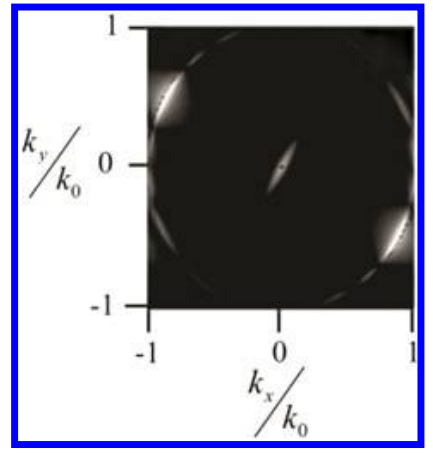

(e)

Fig. 5. (a) Topographic image of a line-like surface defect. (b) LRM image of the direct SPP excitation taking place at the center of the line-like defect, and (c) corresponding calculated image. (d) Fourier space LRM and (e) calculated images. The dotted circle and arrow in (a) indicate the incident Gaussian beam and its polarization, respectively. The solid line in (c) represents the line surface defect.

point-symmetric $k$-pace images [see Fig. $5(\mathrm{e})$ ]. Thus, we showed that the developed numerical approach gives consistent results and can be used to model SPP scattering on more complicated systems such as high-density random arrays of nanoparticles.

\subsection{Random particles arrays: High-density area}

In general, the phenomena related to the regime of SPP multiple scattering are rather complicated and their interpretation is far from being trivial. This is due, at least partially, that the randomness of the interactions yields a large number of scattering events. We studied SPP multiple scattering on a small area (Area1) of the high-density region of nanoparticles or clusters. Figure 6(a) shows intensity distributions where the multiple interference effects are already pronounced. For example, at the center of the image in Fig. 6(a), i.e., within the area of the scatterers, one can appreciate bright and dark regions which are a collection of small and round bright spots similar to those reported as evidence of localized SPPs. ${ }^{17}$ The corresponding Fourier spectrum [see Fig. 6(b)] showed a fingerprint of the excited SPPs (which propagates almost in all possible directions of Area1) and the interference between such excited modes and SPP scattered in all possible directions. In other words, the Fourier spectrum contains a nearly filled circle that corresponds to the well-developed multiple scattering. ${ }^{17}$ Actually, in such SPP scattering regime, two images with a slightly different number of particles and distributions may exhibit distinct total intensity fields. Indeed, a small variation of the particles distributions and/or parameters of the incident light may change significantly the total intensity field distribution. LRM images taken at a different and somewhat rougher surface area (Area2) of the gold film showed a very complicated interference pattern [see Fig. 6(c)]. In fact, it is possible that only a few bright spots should be directly related to the excited and scattered SPPs. For example, if the nanoparticles are very close to each other, the nanoarray is almost symmetric over the extent of the incident beam and therefore cannot scatter efficiently in the axial direction since the incoming propagating vector and the propagating SPP vector are hardly matched. The above mentioned is also exhibited in the Fourier spectrum where a clear SPP fingerprint of the interference between the excited and the scattered SPP's is almost not present [see Fig. 6(d)]. In this context, the corresponding numerical simulations where made for a total area of $27 \times 27 \mu \mathrm{m}^{2}$ in 


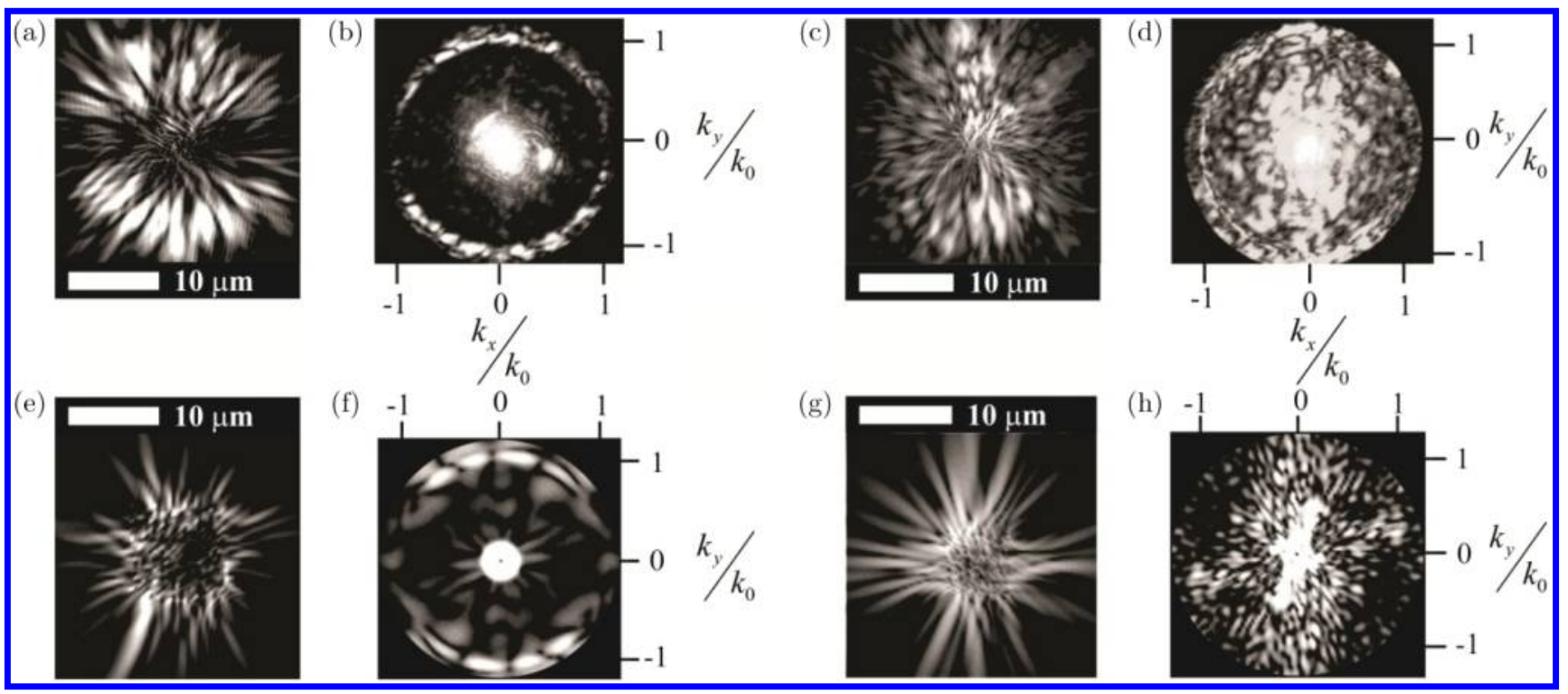

Fig. 6. LRM and calculated images of Area1 (a, e) and Area2 (c, g) sections of nanoparticles illuminated with a free-space excitation wavelength of $633 \mathrm{~nm}$ and $(\mathrm{b}, \mathrm{d}, \mathrm{f}, \mathrm{h})$ Fourier space images of (a, c, e, g), respectively. Incident light polarization is the same as in Fig. 3.

which 100 (representing Area1) and 150 (representing Area2) scatterers were randomly distributed in a central area of $10 \times 10 \mu \mathrm{m}^{2}$ [see Figs. 6(e) and $6(\mathrm{~g})]$. Since we assume that the (point-like) particles are perfectly symmetric and homogeneously distributed in a square area, the calculated $k$-space images present a point-symmetric behavior. It is clearly not the case in the experiments. However, in general, the simulations showed a good agreement with the corresponding above-mentioned experimental case [see Figs. 6(e)-6(h)]. Similar investigations were carried out in another region of the

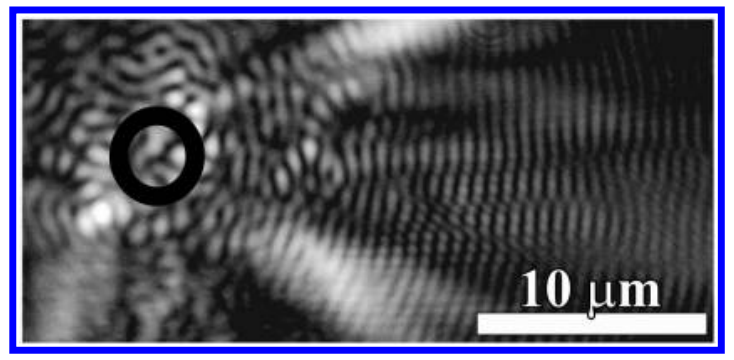

(a)

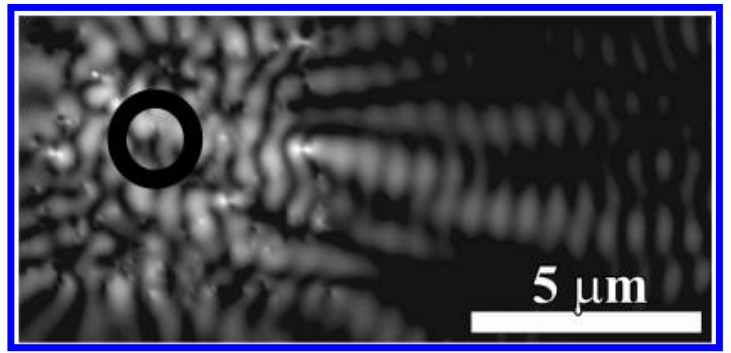

(c)

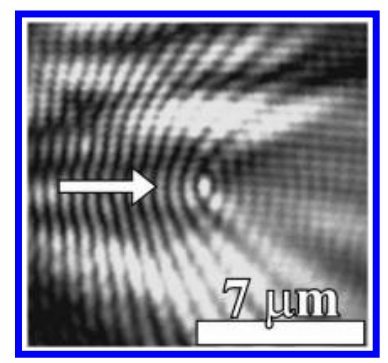

(b)

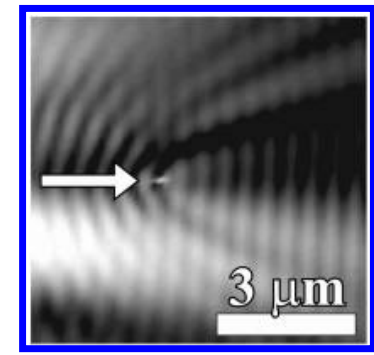

(d)

Fig. 7. (a) LRM and (c) $\left(15 \times 5 \mu \mathrm{m}^{2}\right)$ calculated images of high density of nanoparticles illuminated with a free-space excitation wavelength of $633 \mathrm{~nm}$. (b) LRM and (d) $\left(8 \times 5 \mu \mathrm{m}^{2}\right)$ calculated images of a SPP plane wave elastically scattered. The solid circle in (a) and (c) indicates the incident Gaussian beam. The white arrow in (b) and (d) indicates the SPP incident direction. Incident light polarization is the same as in Fig. 3 . 
random nanoarray that contains the high density of particles. LRM and calculated images have shown considerably less pronounced effects of multiple SPP scattering outside the random structures [see Fig. 7(a) and 7(c)]. Outside the nanoparticle cluster, where only a few scatterers are present, a nearly plane SPP wave is propagating in the specular direction with respect to an imaginary boundary of the region of nanoparticles [see Figs. 7(a) and 7(c)]. Such SPP wave was achieved by using the cluster of nanoparticles randomly distributed over the thin gold film as a mechanism of simultaneous SPP excitation and propagation. Then, far from its excitation origin $(\sim 10 \mu \mathrm{m})$, the SPP behave as a nearly plane SPP wave. A SPP plane wave impinging on a nanoparticle indeed shows a parabolic interference pattern in the resulting total intensity distribution (as long as the elastic scattering is preserved). Figures 7(b) and 7(d) show an LRM and calculated images that correspond to the interference between a SPP mode with a plane phase front (originated outside the scatterer area) and a scattered SPP, due to a nearly isolated nanoparticle, with a cylindrical phase front. The existence of such well-pronounced parabolic interference fringes are the basis of the dipole multiple scattering vectorial model. ${ }^{19}$

\section{SPPs Scattering by Various Nanoparticles}

In the low nanoparticle density region, we often observed regions of nearly isolated nanoparticles lying on the surface and relatively close to each other. We took advantage of those random distributions in order to study near-field interactions between closely spaced particles, particularly effects of SPP refraction. ${ }^{13}$ As mentioned previously, by focusing the illumination beam to a nearly isolated nanoparticle, dipole-like damped radiation can be achieved. On the other hand, a line of nanoparticles can act as a beam splitter ${ }^{10}$ or as a mirror ${ }^{9}$ mostly depending on the inter-particle distance. We generated a dipole-like SPP source which pointed toward a line-like nanostructure [see Figs. 8(a) and 8(b)]. Collective SPP refraction effects were clearly exhibited during the course of SPP propagation. For example, the interaction between the dipole source and the line nanostructure showed a beam splitting effect. Far from its origin $(\sim 10 \mu \mathrm{m})$, the transmitted beam strikes a small cluster of nanoparticles and parabolic interference fringes can be clearly

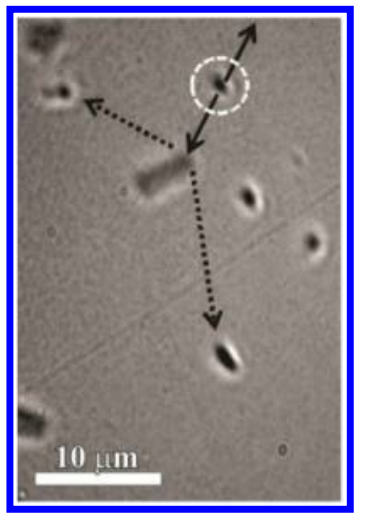

(a)

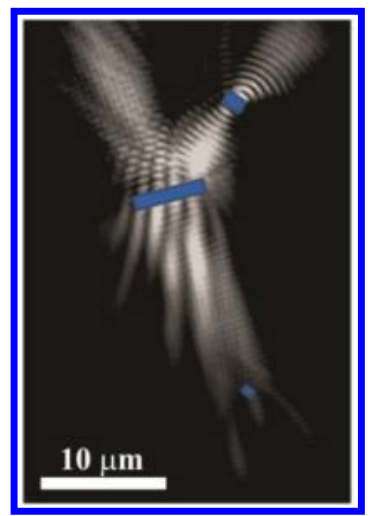

(b)

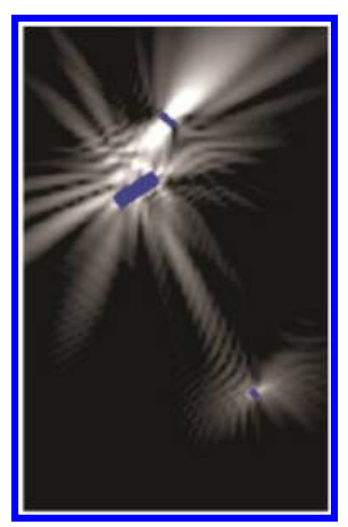

(c)

Fig. 8. SPP refractive effects. (a) Topography, (b) LRM and (c) $\left(10 \times 15 \mu \mathrm{m}^{2}\right)$ calculated images. The dotted circle in (a) indicates the incident Gaussian beam. The arrows in (a) help to guide the eye from the SPP excitation (solid) to the several refraction effects (dotted). The lines in (b) and (c) represent the surface features of (a). Incident light polarization is the same as in Figs. 3(a) and 3(b).

observed in the total intensity distribution. Comparison between numerical and experimental results showed a good correlation [see Figs. 8(b) and $8(\mathrm{c})]$.

\section{Conclusions}

Summarizing, simultaneous SPP excitation and propagation control in random $2 \mathrm{D}$ arrays of nanoparticles have been investigated with the help of LRM imaging. Numerical simulations based on the Green's tensor formalism show a good match with the experimental results. The numerical calculations were carried out by using a relatively simple vectorial dipolar model for multiple SPP scattering $^{25}$ that allows one to explicitly formulate the set of linear equations for the self-consistent field, facilitating 
greatly computer aided design considerations. Experimental results obtained for dipole-like radiation have been presented. The corresponding cross polarized detection has exhibited four extended lobes that are formed only after an initial transition region. Using a line-like surface defect, the experimental feasibility of simultaneous excitation, and propagation of SPP fields was corroborated. For randomly (high-density) situated nanoparticles, the experimental and numerical results of simulations of different scattering regimens and related phenomena have been presented illustrating the interplay between different orders of scattering and SPP phenomena. We demonstrated the possibility to perform interactive refractive effects on a system of particles that are relatively near to each other. Even though the similar properties had already been observed on nanoshaped arrays, we believe that, if properly designed, the above-mentioned nanoparticle systems offer more capabilities for nanophotonics systems integration. In general, theoretical modeling of multiple SPP scattering regimes is quite a challenge in itself, because one has to deal with a large number of scattering events, however, the obtained results reproduced good all the qualitative tendencies found in the experimental study. A detailed comparison between data from experimental measurements and numerical simulations is very difficult to assess quantitatively. For example, in our configuration, the direct evaluation of the optimum SPP coupling efficiency using the vectorial dipolar model is cumbersome and typically omitted, ${ }^{32}$ since it should involve, among other things, a careful analysis of strong particle-surface interactions whose accurate description might require going beyond the framework of dipole scattering approach. We would like to emphasize that the main idea of the proposed experimental approach was in avoiding the usage of additional interfacing elements such as, for example, in-coupling ridges and focusing elements. However, we are clear that even though a certain understanding about multiple SPP scattering phenomena was gained, the outcome of these investigations clearly made calls for more systematic analyses. A statistical study seems to be a different and powerful approach in order to elucidate further in this field. ${ }^{33}$ Based on the results obtained here, we also conclude that a search of new experimental and numerical approaches for plasmonic modes in random mediums remains to be an open problem.

\section{Acknowledgments}

The authors acknowledge financial support from CONACyT project 127589 and scholarship 228959.

\section{References}

1. S. Maier, Plasmonics: Fundamentals and Applications (Springer, New York, 2007).

2. L. Novotny and B. Hecht, Principles of Nano-Optics (Cambridge University Press, New York, 2006).

3. H. Raether, Surface Plasmons, Springer Tracts in Modern Physics, Vol. 111 (Springer, Berlin, 1988).

4. V. M. Agranovich and D. L. Mills (eds.), Surface Polaritons (North-Holland, Amsterdam, 1982).

-5. L. Novotny and S. Stranick, Annu. Rev. Phys. Chem. 57, 303 (2006).

6. A. Diaspro, Optical Fluoresence Microscopy: From the Spectral to the Nano Dimension (Springer, New York, 2011).

-7. A. Hohenau, J. R. Krenn, A. Drezet et al., Opt. Express. 19, 25749 (2011).

-8. C. Garcia, V. Coello, Z. Han et al., Opt. Express. 20, 7771 (2012).

-9. M. U. Gonzales, A. L. Stepanov, J. C. Webber et al., Opt. Lett. 32, 2704 (2007).

10. H. Dittlbacher, J. R. Krenn, G. Shider et al., Appl. Phys. Lett. 81, 1762 (2002).

11. T. Holmgaard, Z. Chen, S. I. Bozhevolnyi et al., Appl. Phys. Lett. 94, 051111 (2009).

12. C. Garcia, V. Coello, Z. Han et al., Appl. Phys. B. 107, 401 (2012).

13. I. P. Radko, A. B. Evlyukhin, A. Boltasseva et al., Opt. Express 16, 3924 (2008).

14. J. B. Khurgin and G. Sun, Appl. Phys. Lett. 94, 221111 (2009).

15. M. I. Stockman, S. V. Faleev and D. A. Bergman, Phys. Rev. Lett. 87, 167401 (2001).

16. L. Dal Negro, N. Feng and A. Gopinath, J. Opt. A: Pure Appl. Opt. 10, 064013 (2008).

-17. V. Coello, Surf. Rev. Lett. 15, 867 (2008).

18. S. I. Bozhevolnyi, V. Volkov and K. Leosson, Phys. Rev. Lett. 89, 186801 (2002).

19. T. Søndergaard and S. I. Bozhevolnyi, Phys. Rev. B. 67, 165405 (2003).

20. V. Coello and S. I. Bozhevolnyi, Opt. Commun. 282, 3032 (2009).

21. I. P. Radko, S. I. Bozhevolnyi, G. Brucoli, L. MartinMoreno, F. J. Garcia-Vidal and A. Boltasseva, Phys. Rev. B. 78, 115115 (2008).

-22. D. S. Kim, S. C. Hohng, V. Malyarchuk et al., Phys. Rev. Lett. 91, 143901 (2003).

-23. E. Devaux, T. W. Ebbsen, J.-C. Webber et al., Appl. Phys. Lett. 83, 4936 (2003). 
24. N. Hartmann, G. Piredda, J. Berthelot et al., Nano Lett. 12, 177 (2012).

25. P. Segovia and V. Coello, Nano 1, 1150003 (2012).

-26. K. Kneipp, H. Kneipp, I. I. R. Dassar et al., J. Phys. Condens. Matter 14, R597 (2002).

27. N. Elizondo, P. Segovia, V. Coello et al., Green synthesis and characterizations of silver and gold nanoparticles, in Green Chemistry - Environmentally Benign Approaches (Intech, New York), 2012).

-28. O. Keller, M. Xiao and S. I. Bozhevolnyi, Surf. Sci. 280, 217 (1993).
29. Z. Li, B. Gu and G. Yang, Phys. Rev. B 55, 10883 (1997).

30. P. Gay-Balmaz and O. Martin, Opt. Commun. 184, 34 (2000).

-31. B. Hecht, H. Bielefeldt, L. Novotny et al., Phys. Rev. Lett. 77, 1889 (1996).

-32. A. B. Evlyukhin, S. I. Bozhevolnyi, A. L. Stepanov et al., Opt. Express. 15, 16667 (2007).

-33. S. I. Bozhevolnyi and V. Coello, Phys. Rev. B. 64, 115414 (2001). 